\title{
Clinical effects of MIPPO technology in patients with anterior ring instability fracture.
}

\author{
Zhi-Hong Wang ${ }^{1 *}$, Ming-Ming Wang ${ }^{2}$, Bin Xu ${ }^{1}$, He-Huan Xia ${ }^{1}$, Jun-Ming Cao ${ }^{1}$, Yong-Sheng Lin ${ }^{1}$, Yi- \\ Peng Yang ${ }^{1}$ \\ ${ }^{1}$ Department of Orthopaedics, the Third Hospital of Hebei Medical University, Shijiazhuang, Hebei, PR China \\ ${ }^{2}$ Department of Orthopaedics, Shi Jia Zhuang Greatwall Hospital, Shijiazhuang, Hebei, PR China
}

\begin{abstract}
Objective: This study aims to discuss the clinical effects of Minimally Invasive Percutaneous Plate Osteosynthesis (MIPPO) technology in patients with anterior ring instability fracture.

Methods: The participants were 76 patients with anterior ring instability fracture who were admitted in our hospital from April 2015 to June 2017. The patients were divided into control $(n=38)$ and treatment groups $(\mathrm{n}=38)$ by using random number table. The control group was treated with improved Stoppa reconstructive plate fixation, whereas the treatment group was administered with MIPPO technology. The operation conditions, Majeed functional improvement effect, comprehensive therapeutic effect, and postoperative complications of the two groups were compared.

Results: The treatment group is significantly superior over the control group in terms of time of operation, intraoperative blood loss, length of stay, and time of fracture healing $(P<0.05)$. The Majeed functional scores of both groups at 1 and 3 months after the operation are significantly higher than those before the operation. In addition, the Majeed functional scores of the treatment group are higher than those of the control group $(\mathbf{P}<\mathbf{0 . 0 5})$. The control group achieves significantly lower clinical efficiency than the treatment group $(\mathbf{P}<\mathbf{0 . 0 5})$. By contrast, the treatment group suffers fewer complications than the control group $(\mathbf{P}<0.05)$.

Conclusions: MIPPO technology significantly affects anterior ring instability fracture. This method is characteristic of small trauma, high safety, feasibility, and quick recovery and is worthy of further clinical popularization.
\end{abstract}

Keywords: Anterior ring instability fracture, MIPPO technology, Clinical effect.

Accepted on December 7, 2017

\section{Introduction}

Anterior ring mainly refers to the part that is formed by the superior ramus of bilateral pubis through the symphysis pubis. The pelvis at this position is extremely weak and easily fractured by external forces [1]. Most fractures are identified as instability fracture, which is difficult to treat and has non-ideal prognosis. The therapeutic effect of conventional treatment is often unsatisfying [2]. Minimally Invasive Percutaneous Plate Osteosynthesis (MIPPO) technology has recently become a common clinical therapy for bone fractures due to its increasing application. Relevant studies have proved the high effect, safety, and reliability of this technique for the treatment of bone fracture [3,4]. However, few studies have been conducted on its application in anterior ring instability fracture. In the present study, the participants were 76 patients with anterior ring instability fracture who were admitted in our hospital from April 2015 to June 2017. Results are introduced in the following text.

\section{Information and Methods}

\section{General information}

The participants were 76 patients with anterior ring instability fracture who were admitted in our hospital from April 2015 to June 2017. Inclusion standards were as follows: agreement with the diagnosis standards of anterior ring instability fracture; diagnosis by pelvis CT and X-ray images; and approval by the Ethics Committee of the hospital. The patients were informed about the research goal and have signed the informed consent. Exclusion standards were as follows: experienced immunity dysfunction, abnormal renal function, coagulation disorders, and serious infection; diagnosed with blood disease, insanity, and unconsciousness; and has incomplete clinical data. The patients were divided into the control $(n=38)$ and treatment groups $(n=38)$ by using the random number table. The control group includes 23 males and 15 females aged from 18 years to 65 y $(42.6 \pm 7.4)$ and had 3 cases of fall, 7 cases of hitting, 12 cases of fall injury, and 16 
cases of traffic accidents. The treatment group includes 24 males and 14 females aged from 19 years to 66 y $(43.5 \pm 7.3)$ and had 1 case of fall, 6 cases of hitting, 14 cases of fall injury, and 17 cases of traffic accidents. Statistical comparison on the general information revealed no significant difference between the two groups $(\mathrm{P}>0.05)$.

\section{Methods}

Preoperative preparation: Prior to operation, all patients have to receive conventional disinfection treatment to the operative region to prepare the preserved skin. General anesthesia was adopted. The patients with pure anterior ring fracture were asked to lie on their back for the operation, whereas those with other types of fracture were requested to be in a floating position for the operation.

The control group was treated by Stoppa reconstructive plate fixation as follows: conventional indwelling urinary catheter, bed hedgehopping on the affected side, and cooperation of pull resetting during operation. A transverse incision was made on the symphysis pubis, and pulling open protection was implemented to the spermatic cord or round ligament of uterus to fully expose the symphysis pubis. Special machines were used to restore and separate the symphysis pubis. Internal steel plate fixation was adopted. Fracture position and incision deviation were used as the reference. Femoral nerve, iliopsoas, and arteries and veins outside the ilium do not need to be exposed. The reconstructed steel plates at preflex were placed below the true pelvis through the undermining dissection of pubic ramus. Fluoroscopic examination was performed by using the C-type arm X-ray fluoroscopic machine to confirm the restoration condition. Finally, crossing acetabulum fixation was implemented. The treatment group was administered with MIPPO technology: $2-4 \mathrm{~cm}$ long transverse and 2-3 cm long inclined incisions were made at the symphysis pubis and anterior superior spine, respectively. The musculus rectus abdominis at the symphysis pubis was conventionally separated along the incision on anterior superior spine until reaching the aponeurosis of obliquus externus abdominis. Passivity analysis of tissues was subsequently conducted. The subcutaneous channel was constructed by using the musculus obliquus externus abdominis. Locations of far-end spermatic cord and near-end nervus cutaneus femoris lateralis were determined. Steel plates were inserted into the near end from the far end of subcutaneous channel. Bolts were installed at near- and far-end cortexes. In the bone surface of outer sides of steel plates, pressure was increased, and the bolts were input and locked. After the steel plates were placed and locked successfully, the operation cavity was rinsed, and the drainage tube was indwelled. The incision was closed, and the operation was accomplished.

Postoperation: drainage tube was indwelled conventionally 2-4 d after the operation. The patients were asked to lie on their back and were offered with intravenous drip of antibiotics. Anti-freezing of low molecular heparin was offered, and nutritional supports were enhanced. Functional rehabilitation training shall be conducted as early as possible, and pertinence measures shall be used for patients with complications.

\section{Observation indexes}

Operation conditions mainly include the time of operation, intraoperative blood loss, time of fracture healing, and Length of Stay (LOS). The Majeed functional scores before the operation and at 1 and 3 months after the operation were evaluated. Post-operative complications, such as nervus vascularis damage, wound infection, and Deep Vein Thrombosis (DVT), were discussed during the follow-up.

\section{Therapeutic evaluation standards}

Therapeutic effect was evaluated according to the Majeed functional scores (100 scores) as follows: excellent, 85-100; good, 70-84; moderate, 55-69; and poor, $<54$. The therapeutic effect was the sum of the proportions of excellent and good scores.

\section{Statistical analysis}

Statistical analysis of observation data was conducted by SPSS22.0. Operation conditions and Majeed functional scores were described as $\overline{\mathrm{x}} \pm \mathrm{s}$ and examined by t-test. The therapeutic effect and occurrence rate of complications were described as $\%$ and examined by $\chi^{2}$-test. $\mathrm{P}<0.05$ indicates statistically significant difference between the two groups.

\section{Results}

\section{Operation conditions}

The treatment group presents significantly shorter time of operation, time of fracture healing, and LOS but higher intraoperative blood loss than the control group $(\mathrm{P}<0.05)$ (Table 1).

Table 1. Comparison of operation conditions between the two groups $\bar{x} \pm s$.

\begin{tabular}{|c|c|c|c|c|}
\hline Groups & Time of operation (min) & Intraoperative blood loss (ml) & $\operatorname{LOS}(d)$ & Time of fracture healing (d) \\
\hline Treatment $(n=38)$ & $241.16 \pm 15.14$ & $384.16 \pm 23.61$ & $16.63 \pm 5.17$ & $31.16 \pm 5.20$ \\
\hline Control $(n=38)$ & $274.49 \pm 18.25$ & $425.57 \pm 28.43$ & $21.16 \pm 7.43$ & $48.57 \pm 6.84$ \\
\hline $\mathrm{t}$ & 10.5184 & 8.3853 & 3.745 & 15.5929 \\
\hline$P$ & 0 & 0 & 0.0003 & 0 \\
\hline
\end{tabular}




\section{Majeed functional scores before and after the operation}

The Majeed functional scores of the two groups at 1 and 3 months after the operation increased to some extent than that before operation. The Majeed functional scores of the treatment group are significantly higher than those of the control group $(\mathrm{P}<0.05)$ (Table 2$)$.

Table 2. Comparison of the Majeed functional scores of the two groups before and after treatment ( $\bar{x} \pm s$, scores).

\begin{tabular}{llll}
\hline Groups & $\begin{array}{l}\text { Before } \\
\text { operation }\end{array}$ & $\begin{array}{c}\text { the } \\
\text { At } 1 \text { month after } \\
\text { the operation }\end{array}$ & $\begin{array}{l}\text { At } \mathbf{3} \text { months after } \\
\text { the operation }\end{array}$ \\
\hline Treatment $(n=38)$ & $38.53 \pm 3.61$ & $72.49 \pm 4.86$ & $89.96 \pm 8.41$ \\
\hline Control $(n=38)$ & $38.79 \pm 3.84$ & $60.53 \pm 4.24$ & $75.25 \pm 6.77$ \\
\hline$t$ & 0.3691 & 13.8769 & 10.1960 \\
\hline$P$ & 0.7127 & 0.0000 & 0.0000 \\
\hline
\end{tabular}

\section{Clinical therapeutic effect}

The therapeutic effect on the control group is $81.6 \%$, which is significantly lower than that on the treatment group $(97.4 \%)$ $(\mathrm{P}<0.05)$ (Table 3).

Table 3. Comparison of the therapeutic effect between the two groups $(n(\%))$.

\begin{tabular}{llllll}
\hline Groups & Excellent & Good & Moderate & Poor & $\begin{array}{l}\text { Therapeut } \\
\text { ic effect }\end{array}$ \\
\hline $\begin{array}{l}\text { Treatment } \\
(n=38)\end{array}$ & $21(55.3)$ & $16(42.1)$ & $1(2.6)$ & $0(0.0)$ & $37(97.4)$ \\
\hline Control $(n=38)$ & $17(44.7)$ & $14(36.8)$ & $5(13.2)$ & $2(5.3)$ & $31(81.6)$ \\
\hline$X^{2}$ & & & & 5.0294 \\
\hline$P$ & & & & 0.0249 \\
\hline
\end{tabular}

\section{Complications after the operation}

The occurrence rates of complications of the control and treatment groups after the operation are $26.3 \%$ and $7.9 \%$, respectively, showing a statistically significant difference $(\mathrm{P}<0.05)$ (Table 4).

Table 4. Comparison of the complications between the two groups after the operation (n (\%)).

\begin{tabular}{|c|c|c|c|c|c|}
\hline Groups & $\begin{array}{l}\text { Wound } \\
\text { infection }\end{array}$ & $\begin{array}{l}\text { Vascular } \\
\text { damages }\end{array}$ & nerve & DVT & Complications \\
\hline $\begin{array}{l}\text { Treatment } \\
(n=38)\end{array}$ & $2(5.3)$ & $0(0.0)$ & & $1(2.6)$ & $3(7.9)$ \\
\hline $\begin{array}{l}\text { Control } \\
(n=38)\end{array}$ & $7(18.4)$ & $1(2.6)$ & & $2(5.3)$ & $10(26.3)$ \\
\hline$x^{2}$ & & & & & 4.5470 \\
\hline $\mathrm{P}$ & & & & & 0.0329 \\
\hline
\end{tabular}

\section{Discussion}

The pelvis is mainly composed of anterior and posterior rings. The anterior ring is the most vulnerable region in the whole pelvis and has the highest damage rate [5]. With the recent booming traffic and construction, the occurrence rate of anterior ring fracture increases gradually and has high disability rate and lethality and poor prognosis. Scientific and effective symptomatic treatment as early as possible is necessary to increase the clinical effect and improve the living quality of patients [6]. Treating instability fracture is difficult because it is the major type of anterior ring fracture. Conventional means, traction restoration, steel plate internal fixation, and plaster immobilization cannot effectively restore and fix the fracture [7]. These methods easily cause fracture malunion, affect the normal physiological structure of the pelvis, increase pains, and influence the survival quality and physical and psychological health of the patients.

Although the improved Stoppa reconstructive plate fixation can be simply operated, it has limited application ranges and high occurrence rate of complications, thus failing to achieve satisfying treatment for all patients [8]. The development and application of MIPPO technology provide a new alternative for the clinical treatment of bone fractures [9]. Clinical practices have confirmed that MIPPO technology can meet the requirements, such as minimally invasive, simple operation, and few complications, of fracture treatment. However, further explorations and evaluation of its therapeutic effect to anterior ring instability fracture are still needed [10].

In this study, the two groups showed significant differences in terms of time of operation, intraoperative blood loss, LOS, and time of fracture healing $(\mathrm{P}<0.05)$. The results indicate that MIPPO technology can significantly shorten the operation and recovery time, reduce blood loss, and increase safety to anterior ring instability fracture. At 1 and 3 months after the operation, the treatment group had significantly higher Majeed functional scores but lower occurrence rate of complications than the control group $(\mathrm{P}<0.05)$. Hence, MIPPO technology can relieve pains, improve the living quality (e.g., standing and walking), reduce the occurrence rate of complications, and facilitate functional recovery, thus further improving prognosis and clinical effects.

\section{Conclusion}

In summary, MIPPO technology significantly affects anterior ring instability fracture. This method is characteristic of small trauma, high safety, feasibility, and quick recovery and is worthy of further clinical popularization.

\section{References}

1. Ma W, Xu N, Hu Y, Li G, Zhao L, Sun S, Jiang W, Liu G, $\mathrm{Gu}$ Y, Liu J. Unstable atlas fracture treatment by anterior 
plate C1-ring osteosynthesis using a transoral approach. Eur Spine J 2013; 22: 2232-2239.

2. Kawaoka T, Hanakita J, Takahashi T, Watanabe M. Three case reports of donor site iliac bone fracture as a complication of anterior cervical fixation surgery. NMC Case Rep J 2015; 2: 109-113.

3. Khamesipour F, Tajbakhsh E. Analyzed the genotypic and phenotypic antibiotic resistance patterns of Klebsiella pneumoniae isolated from clinical samples in Iran. Biomed Res India 2016; 27: 1017-1026.

4. Rommens PM, Ossendorf C, Pairon P, Dietz SO, Wagner D, Hofmann A. Clinical pathways for fragility fractures of the pelvic ring: personal experience and review of the literature. J Orthop Sci 2015; 20: 1-11.

5. Li ZY, Yang ZC. Ticagrelor-oxcarbazepine interaction during anti-epileptic therapy in children. Lat Am J Pharm 2017; 36: 1930-1932.

6. Ji F, Tong D, Tang H, Cai X, Zhang Q, Li J, Wang Q. Minimally invasive percutaneous plate osteosynthesis (MIPPO) technique applied in the treatment of humeral shaft distal fractures through a lateral approach. Int Orthop 2009; 33: 543-547.

7. Hafiz TA, Mubaraki MA. The potential role of Ziziphus spina-christi leaf extracts against Plasmodium berghei- induced liver and spleen injury. Biomed Res India 2016; 27: 1027-1032.

8. Zhao L, Yang $\mathrm{P}$, Zhu L, Chen A. Minimal invasive percutaneous plate osteosynthesis (MIPPO) through deltoid-pectoralis approach for the treatment of elderly proximal humeral fractures. BMC Musculoskelet Disord 2017; $18: 187$.

9. Li M, Zhang XF, Sun G, Bai Y, Liu CH. Co-administration risk between regorafenib and irinotecan during the therapy of lung cancer patients. Lat Am J Pharm 2017; 36: 1796-1800.

10. Gupta A, Anjum R, Singh N, Hackla S. Outcome of distal both bone leg fractures fixed by intramedulary nail for fibula and MIPPO in Tibia. Arch Bone Jt Surg 2015; 3: 119-123.

\section{${ }^{*}$ Correspondence to}

Zhi-Hong Wang

Department of Orthopaedics

The Third Hospital of Hebei Medical University

PR China 